\title{
Rescuing Corrupted Gel Files from Model 377 and 373 DNA Sequencers
}

BioTechniques 22:1162-1163 (June 1997)

Kevin M. O'Brien, John W. Fondon III, Glen A. Evans and Harold R. Garner

University of Texas, Southwestern Medical

Center, Dallas, TX, USA

\begin{abstract}
Automated DNA sequencing requires the intensive use of computers to handle the large amount of data taken. When a computer failure occurs and the data are no longer accessible, all the expense and effort that went into the sequencing experiment is lost. By using the data storage architecture of Macintosh ${ }^{\circledR}$ computers to our advantage, we may prevent this loss in the case of automatic sequencers from PE Applied Biosystems. The software required to allow the experimenter to do this has been written and is available free of charge.
\end{abstract}

To our knowledge, the most widely used automated DNA sequencers in the world are the Model 377 and 373 DNA Sequencers (PE Applied Biosystems, Foster City, CA, USA). Both of these devices store the data they collect into gel files on Macintosh ${ }^{\circledR}$ computers. Occasionally, a gel file will become corrupted after all the data were collected. The PRISM $^{\text {TM }}$ Sequencing Analysis Software (PE Applied Biosystems) cannot open a corrupted gel file. Thus, if the gel file cannot be repaired, it is lost entirely. Our experience places the gel file corruption rate at approximately $0.5 \%$, with a loss of roughly $\$ 230$ per failure; this assumption is calculated based on a 36-lane experiment and noting that the PE Applied Biosystems 1994 Product Information Catalog lists a 100 -reaction kit at a price of $\$ 625$ (another $\$ 5$ is added to cover the cost of the gel mixture). Note that labor costs are not included in this analysis.

We wrote a simple program to rescue the data from a corrupted gel file. The Macintosh computer has the following file architecture: all files consist of two data storage areas called forks; the data fork and the resource fork. Apart from a few conventions, it is entirely up to the original programmer as to what piece of information will be stored in which fork. For instance, some word processors store a document's text in the data fork and the document's formatting information in the resource fork (1). In the case of gel files from PE Applied Biosystems, all the trace information is stored in the data fork, as is the so-called "EPT" (electric field, power and temperature) information (i.e., fluctuations during the run in operating voltage, temperature, current and power) and the gel image you see on your computer screen. Among other things, the resource fork contains the sample names, written to it from the sample sheet. It has been found that the majority of the gel file corruptions are due to an error somewhere in the resource fork (J.W. Fondon, unpublished), which means the sequence information necessary to create trace files and call bases is still perfectly "healthy", but the file simply cannot be opened properly by the PE Applied Biosystems's sequencing analysis software. It was possible to write a simple program that transfers the data fork from the corrupted gel file to an uncorrupted gel file. The uncorrupted gel file's data fork will be overwritten, but its resource fork can then be used to open and examine the no-longer-corrupted gel file's samples.

This program, Gel_Rescuer.c, is available free of charge at the World Wide Web (WWW) site: http://mcdermott. swmed.edu/gestec/informatics/gestec_software. Also 
available are a complete set of instructions and a copy of the source code. The program's executable file should be placed in the same folder on your Macintosh as both the corrupted gel file and an uncorrupted gel file that can be overwritten. As the program is presently written, neither of the two gel file's names can have any spaces in them. Note also that since the sample names are resident in the resource fork, the names of the sample files will be incorrect after running Gel_Rescuer.c. The user will have to manually change the trace file names to fit the original gel file. Gel_Rescuer.c was written with Metrowerks Code Warrior on a UMax SUPERMAC PPC clone (UMax Technologies, Santa Clara, CA, USA); however, it has since been run successfully on a variety of other Macintosh computers.

Finally, PE Applied Biosystems provides several means of gel file manipulation in PRISM Sequencing Analysis Software, all of which are utilities under the program GelDocII. One of these, Repair Resources (-39), is in many cases able to repair the resource forks of corrupted gel files. However, its use is limited to gel files generated by a Model 373 Sequencer (2). The program Gel_Rescuer.c can be applied not only to gel files created by Model 373 Sequencer, but also to files created by a Model 377 Sequencer (and presumably to a Model $310)$.

\section{ACKNOWLEDGMENT}

Grant support for this work has been provided by NHGRI Grant No. HG00202-07 and the Department of Energy.

\section{REFERENCES}

1.Mark, D. and C. Reed. 1992. Macintosh C Programming Primer, Inside the Toolbox Using THINK C, Vol. I, 2nd ed, p. 40. Addison-Wesley, Menlo Park, CA.

2.PE Applied Biosystems. GelDocII, Appendix E, p. E-1-E-3. In DNA Sequencing Analysis Software User's Manual, Part Number 904436, Rev. A. Foster City, CA.

Received 3 April 1997; accepted 3 April 1997.

Address correspondence to:

Kevin M. O'Brien

Eugene McDermott Center for Human Growth and Development

The University of Texas, Southwestern Medical Center

5323 Harry Hines Blvd.

Dallas, TX 75235-8591, USA

Internet: kevin@mcdermott.swmed.edu orobrien@utarnw.uta.edu 